\title{
Context-Aware Cyber-Physical Worker Assistance in Industrial Systems: A Human Activity Recognition Approach
}

\author{
Elisa Roth \\ Institute for Manufacturing \\ University of Cambridge \\ Cambridge, United Kingdom \\ er542@cam.ac.uk
}

\author{
Mirco Möncks \\ Institute for Manufacturing \\ University of Cambridge \\ Cambridge, United Kingdom \\ mm2393@cam.ac.uk
}

\author{
Thomas Bohné \\ Institute for Manufacturing \\ University of Cambridge \\ Cambridge, United Kingdom \\ tmb35@cam.ac.uk
}

\author{
Luisa Pumplun \\ Information Systems \\ Technische Universität Darmstadt \\ Darmstadt, Germany \\ pumplun@is.tu-darmstadt.de
}

\begin{abstract}
The increasing demand for product customisation is leading to higher complexities within industrial systems. This imposes new challenges for the workforce. One way to support operators' productivity may be context-aware, human-centred cyber-physical assistance systems. Human Activity Recognition (HAR) is a promising approach to enable context-awareness. However, standardised approaches to integrate HAR into existing industrial environments are rare. Particularly, there is a lack of available datasets of industrial activities. Moreover, comparative studies of inertial and visual HAR approaches are still rare. This work therefore proposes Methods-Time Measurement (MTM) as a standardised foundation for creating an industrial activity dataset. Subsequently, five different machine learning algorithms are tested for their recognition performance based on the dataset captured with an inertial sensor suit and an RGB-D sensor. A proof-of-concept is delivered for both sensor categories applied to the scope of 18 MTM-1 activities, whereas inertial data outperformed depth data. K-Nearest Neighbour and Bagged Tree algorithms revealed the best classification accuracy results in this context.
\end{abstract}

\section{Keywords-Cyber-Physical Assistance, Context-aware systems, Human Activity Recognition, Methods-Time Measurement, Industrial Activity Dataset}

\section{INTRODUCTION}

Industrial organisations are facing a need for change due to increasingly dynamic framework conditions, particularly decreasing product lifecycles and increasing product variability [1]. These ultimately lead to a higher industrial complexity, imposing new challenges in terms of increasing product and process knowledge to the workforce. However, there is a discrepancy between most workers' actual performance capacity and proficiency, and the task requirements of evolving Cyber-Physical Production Systems (CPPS) [2]. CPPS generally describe the amalgamation of physical and virtual entities that closely interact within a production system [3]. Researchers state that Cyber-physical assistance systems (CPAS) have the potential to provide a control function to CPPS, as they balance increasing operative requirements with individual human capabilities [2]. Assistance systems should be human-centred technological systems that support employees by giving them real-time feedback, and flexible acting recommendations to secure and improve processes. The success of CPAS partly depends on how accurate technologies can recognise context in order to provide adaptive feedback on a particular sequence of tasks, ergonomics, or use of correct parts and tools. Context-aware, human-centred CPAS have the potential to close the current gap towards closed feedback loops in fully connected CPPS, as information about critical human activities such as quality checks or maintenance is often lacking. Human Activity
Recognition (HAR) is a promising approach to enable context-awareness within CPAS. HAR has the potential to be independently retrofitted into existing industrial environments, even in those with a low level of automation [4]. However, to develop effective HAR-based CPAS requires the integration of standardised approaches to increase industrial applicability [5]. Therefore, this work strives to identify how established standardised methods for manufacturing work measurement, e.g. Methods-Time Measurement, can be applied. While different hardware and software approaches based on sensors and machine learning algorithms exist, no prior research, to the best of our knowledge, has directly compared inertial and vision-based sensors in an industrial context. Moreover, human activity datasets as a foundation for HAR in industrial systems are still rare. This project strives to experimentally test the potential of inertial and visual sensor systems, and further test different algorithmic approaches for robust recognition of MTM-based industrial tasks. In so doing, we created a multimodal dataset with the scope of 18 activities available for future research.

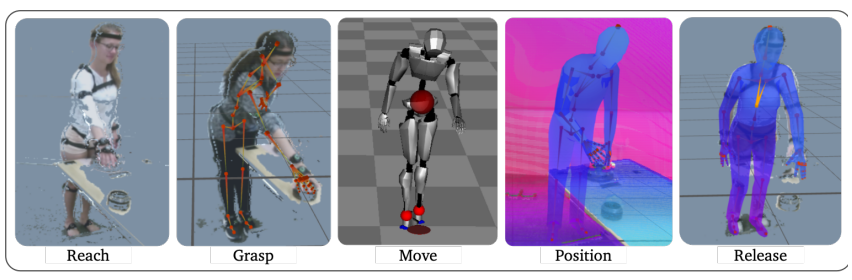

Fig. 1. Exemplary MTM activities from the IfM-CHLab2019 dataset in RGB, RGB+skeleton, avatar, and depth+skeleton representations (from left to right).

\section{RELATED WORK}

\section{A. Cyber-Physical Assistance Systems}

The increasing complexity of production systems demands an enhanced skill set from industrial workers. This ranges from flexibility to operate on a multitude of different processes to complex troubleshooting. Assistance systems promise a way to balance the discrepancy between most workers' actual performance capacity and proficiency, and the increasing task requirements of evolving CPPS [2]. This is especially relevant for low-volume, high-complexity industrial systems where automation will be neither technologically nor economically feasible in the foreseeable future. Context-awareness is crucial for an effective support of human workers [6]. To enable context-awareness in-theloop requires data about actual human activities performed within CPS [7]. However, these data are still missing. Until now, a user typically has to proactively confirm that she accomplished a task [8], but no automatic feedback is 
provided back to the system if a user actually performed the tasks correctly and completely. Thus, there will always be an inherent discontinuity in CPPS, which leads to suboptimal (re)configuration of processes, assistance design, and subsequent errors. Therefore, CPAS need to be automatically synchronised with the operator's activities, without requiring manual feedback. The correct sequence of assembly steps, the adequate use of workpieces, tools, material, and other equipment should be tracked with the help of cameras and other sensors in real-time [5].

\section{B. Human Activity Recognition}

Human Activity Recognition aims to automatically classify activities using data acquired from sensing modalities. To date, HAR has been primarily applied to elderly care, autonomous driving, gaming, or public surveillance. The established procedure in HAR research includes the selection of sensors, the design and capturing of a dataset, the application of pre-processing algorithms, and lastly classification algorithms [9].

\section{1) Sensors for Human Activity Recognition}

The most common sensor categories in HAR are inertial and vision-based sensors [9-10]. Micromachined inertial sensors can measure angular velocities, and linear accelerations of objects relative to known starting points. Inertial approaches in HAR primarily use Inertial Measurement Units (IMUs). IMUs combine gyroscopes, accelerometers, and often magnetometers. For HAR, IMUs are directly worn by humans. However, IMUs suffer from drift due to thermo-mechanical noise, flicker noise, temperature-effects, or calibration error [11]. Apart from drift, IMUs also face constraints in energy consumption based on communication and processing [12]. Nevertheless, inertial sensors have delivered promising accuracy results for HAR [13]. In comparison with vision-based sensors, no line of sight is needed between the subject and the signal receiver.

Vision-based approaches to HAR use different kinds of cameras or range sensors. The cost of depth sensors has reasonably decreased since early HAR approaches, while performance has increased [10]. This is one reason why the current focus in HAR research has shifted from 2D RGB towards $3 \mathrm{D}$ data. $3 \mathrm{D}$ data can be obtained by various distance sensing methods, sometimes in a combined manner. Common methods are stereo vision, motion capture, time-offlight, and structured light [13]. Even if vision based HAR approaches experienced notable advances in the last decade, it remains a technologically demanding task. According to [10], four main aspects are particularly challenging: (1) lowlevel challenges (e.g. occlusion, illumination), (2) viewpoint invariance, (3) scale invariance, and (4) intraclass variability and interclass similarity. Despite visual data processing is computationally more complex and thus more expensive than inertial data, its popularity in HAR increases rapidly [12].

\section{2) Algorithmic Considerations in Human Activity Recognition}

The main steps towards HAR identified by previous research are data pre-processing, segmentation, feature extraction, dimensionality reduction, and classification [13]. Most HAR systems face practical constraints in terms of power supply and computing costs. Therefore, it is essential to conduct dimensionality reduction by extracting certain features which have a high ability to describe the activity of interest [16]. An overview of feature extraction can be found in [9] and [10]. Body model features have shown great potential for both inertial and vision-based sensors. Moreover, Principal Component Analysis (PCA) is popular as a method for dimensionality reduction [17].

In general, classification approaches can be divided into two main groups: supervised and unsupervised machine learning (ML). Supervised ML requires input which is fully labelled with its respective classes. In HAR mostly supervised algorithms are used. Template-based similarity metrics, temporal probabilistic models [18], discriminative approaches, decision trees [19], and ensemble classifier revealed good performances for HAR. However, no one-fitsall technique exists. Researchers have to balance computational resources and recognition performance for a specific research interest.

\section{3) Human Activity Recognition in Industrial Systems}

Early approaches of HAR in industry were made by the wearIT@work project [18]. This project made strides towards a successful application of HAR in training environments of an automotive manufacturer. Nevertheless, the applied technologies cannot be considered as state-of-theart anymore. More recently, [6] and [20] explored visionbased HAR approaches in order to advance human-robot interaction. Decent classification accuracy results could be achieved for four different human activities, such as lowgranular tasks ("general assembly") and high-granular tasks e.g. hand movements. The best result was achieved by Support Vector Machines (RBF 81\%, linear 77\%) [20]. In contrast, another project explored wearable sensors for the recognition of a single pick-and-place task [21]. They applied a Hidden Markov Model supervised learning approach (96\% accuracy). However, the datasets in [6], [21] were captured with only one participant and are thus prone to bias. Moreover, the activity selection was not methodologically derived in previous studies, so a standardised approach to HAR in industrial systems remains missing. In summary, previous industry related HAR approaches have made progress but suffer from four weaknesses: (1) the datasets are not publicly available for future research, (2) direct comparisons of inertial and visual sensors are lacking, (3) variability in tasks and participants is limited, and (4) the studies did not build on a standardised approach towards HAR.

\section{Methods-Time Measurement}

Human work is a main pillar in the conception of success factors in industrial engineering research [22]. Industrial tasks can be conceptualised by following systems of predetermined times for production planning [5]. Especially the Methods-Time Measurement system evolved to a worldwide standard [23]. The method states that tasks execution time depends on the chosen working method. MTM enables the analysis of human motion sequences with respect to a current and a desired status. Apart from motional elements (e.g. grab, position), the system also allows the integration of cognitive functions (e.g. identify). This enables the estimation of task execution times. The result of MTM 
analyses are structured motion sequences aggregated from basic motions.

Within the foundational module MTM-1, basic manufacturing motion elements from three main categories were identified [24], see Figure 2. In assembly, frequently performed elementary movements of the hand-arm-system are reaching, grasping, moving, positioning, and releasing. Further, eye functions are essential in quality assurance within assembly. With regard to ergonomics, full body movements with a tilt of the body axis are also valuable to evaluate. MTM-1 is the foundation for more complex MTM systems and has thus the potential to serve as a database of adaptive, combinable motion modules. Existing studies have solely focused on the potential of MTM to plan the need for digital assistance [5]. So far, no study has addressed how MTM elements can be explicitly used to create standardised datasets as a database foundation for context-aware CPAS.

\begin{tabular}{|c|c|c|c|c|}
\hline \multicolumn{5}{|c|}{ Basic MTM-1 Motions } \\
\hline \multicolumn{2}{|c|}{ Motions of the hand-arm system } & Eye functions & \multicolumn{2}{|c|}{ Full body, leg, and foot motions } \\
\hline \multirow{5}{*}{ 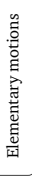 } & Reach $R$ & Eye travel $E$ & \multirow{2}{*}{ 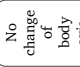 } & Foot motion $F M$ \\
\hline & Grasp $G$ & Eye focus $E$ & & Leg motion $L M$ \\
\hline & Move $M$ & & \multirow{3}{*}{ 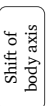 } & Side step SS \\
\hline & Position $P$ & & & Body turn $B T$ \\
\hline & Release $R L$ & & & Walk $W$ \\
\hline \multirow{3}{*}{ 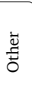 } & Apply pressure $A P$ & & \multirow{4}{*}{ 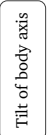 } & Bend and arise \\
\hline & Disengage $D$ & & & toop and arise \\
\hline & Turn $T$ & & & Knee and arise \\
\hline & & & & it and stand up \\
\hline
\end{tabular}

Fig. 2. Basic MTM-1 Motions [25]

\section{SEnsor Testbed And Dataset}

In order to close the research gaps identified in the previous section, we captured a multimodal dataset of 19 participants performing 18 MTM-based industrial activities. For the proposed activity spectrum, the Microsoft Kinect v2 RGB-D sensor was selected as a proven device in HAR studies [9-10]. Further, we used an inertial motion capturing system (Perception Neuron PRO) due to its success in related fields such as occupational ergonomics [26]. The inertial system contains 17 IMUs. The full setup can be seen in Figure 3 .

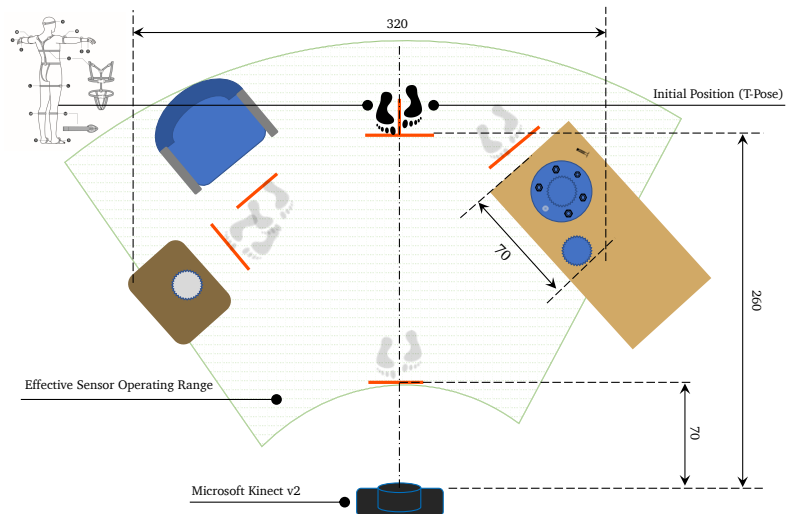

Fig. 3. Visualisation of the experimental setup including the effective sensor operating ranges and relevant distance measures for an enhanced setup replicability (not true to scale)

\section{A. Data Collection}

Our dataset (IfM-CHLab2019) covers 18 of the 19 MTM1 movements [25]. The activity apply pressure was omitted because the selected sensors are inherently not capable of measuring pressure. Those MTM-1 sub-activities are the foundation of most task compositions that can possibly occur in industrial systems and could hence enable the modular development of context-aware worker assistance systems. This assumption is made based on the high level of detail the MTM method includes, and further product information (e.g. from CAD models, assembly manuals, or manufacturing bill of materials) could be integrated too [5].

Depending on the duration of an activity the participants performed the activities with 1 to 5 repetition to achieve a balanced and sufficiently large dataset [9]. For a benchmarking dataset it is essential that there is a broad variation in inherent characteristics of the participants. This will ensure that the HAR system is less biased and later on applicable to new individuals without requiring additional training data [27]. As shown in Table I, the dataset is heterogenous as the participants vary in age, cultural background, gender, and physical constitution. The activities were based on the assembly of a real clutch.

Before the data collection, we successfully sought ethical approval from the University of Cambridge's ethics commission. The detailed explanation of the tasks where derived from established MTM standards [25]. Exemplary extracts of the 18 MTM-1 activities are documented in Figure 4.

The data that support the findings of this study are available from the corresponding lead author upon reasonable request.

TABLE I. SUMMARY OF THE IFM-CHLAB2019 DATASET

\begin{tabular}{|c|c|}
\hline Characteristics & IfM-CHLab2019-Roth \\
\hline Date & $21 / 07 / 2019$ - 23/07/2019 \\
\hline Description & $\begin{array}{l}\text { MTM-based human activities in manufacturing captured by depth and } \\
\text { inertial sensors }\end{array}$ \\
\hline Institution & $\begin{array}{l}\text { University of Cambridge, Institute for Manufacturing, Cyber-Human } \\
\text { Lab }\end{array}$ \\
\hline Scope & 19 (18) participants $\times 18$ activities $\times(1-5)$ repetitions $\times 2$ sensors \\
\hline Variability within sample & $\begin{array}{l}\text { Age range } 16 \text { - } 35 \text { years, } 9 \text { female } / 10 \text { male, height range } 160 \mathrm{~cm} \text { - } \\
191 \mathrm{~cm}\end{array}$ \\
\hline $\begin{array}{l}\text { Frames total (pre-processed } \\
\text { data) }\end{array}$ & RGB-D 80.374, Inertial measurement suit 350.779 \\
\hline Sensors & $\begin{array}{l}\text { RGB-D camera ( } 30 \text { frames per second); Inertial measurement suit } \\
(120 \mathrm{~Hz})\end{array}$ \\
\hline Activities & $\begin{array}{l}18 \text { based on MTM- } 1 \text { ( } 7 \text { hand-arm motions, } 2 \text { eye functions, } 9 \text { full } \\
\text { body motions) }\end{array}$ \\
\hline Extractable rawdata types & $\begin{array}{l}\text { RGB-D: coordinates, velocity, acceleration; Inertial measurement } \\
\text { suit: displacement, rotation }\end{array}$ \\
\hline Orientation & Cartesian Coordinate System \\
\hline
\end{tabular}

\section{B. Data Pre-processing}

Firstly, the RGB-D data underwent background subtraction. Afterwards both sensor data streams were manually segmented into areas containing the activities of interest. In this process, the IMU data for one participant had to be eliminated as the calibration was not done correctly. The RGB-D data were further processed by eliminating jitter. Both raw data sets were reorganised into the same data structure to allow for comparability within the classification performance. The developed pre-processing algorithm 
followed a proposed logic introduced in [28]. This algorithm allows for an input generation suitable for various supervised machine learning algorithms, including deep neural networks. Moreover, the algorithm enables the adaptive selection of activities, participants, body joints, and input modalities to evaluate different research interests. Firstly, the raw joint data for each frame in the selected input mode (coordinates, velocity, acceleration) is extracted in vector format. In the next step, the data underwent normalisation. Subsequently, the data were aggregated participant- and activity-wise into a matrix-form. Lastly, the algorithm assigned activity labels to the data of each frame.

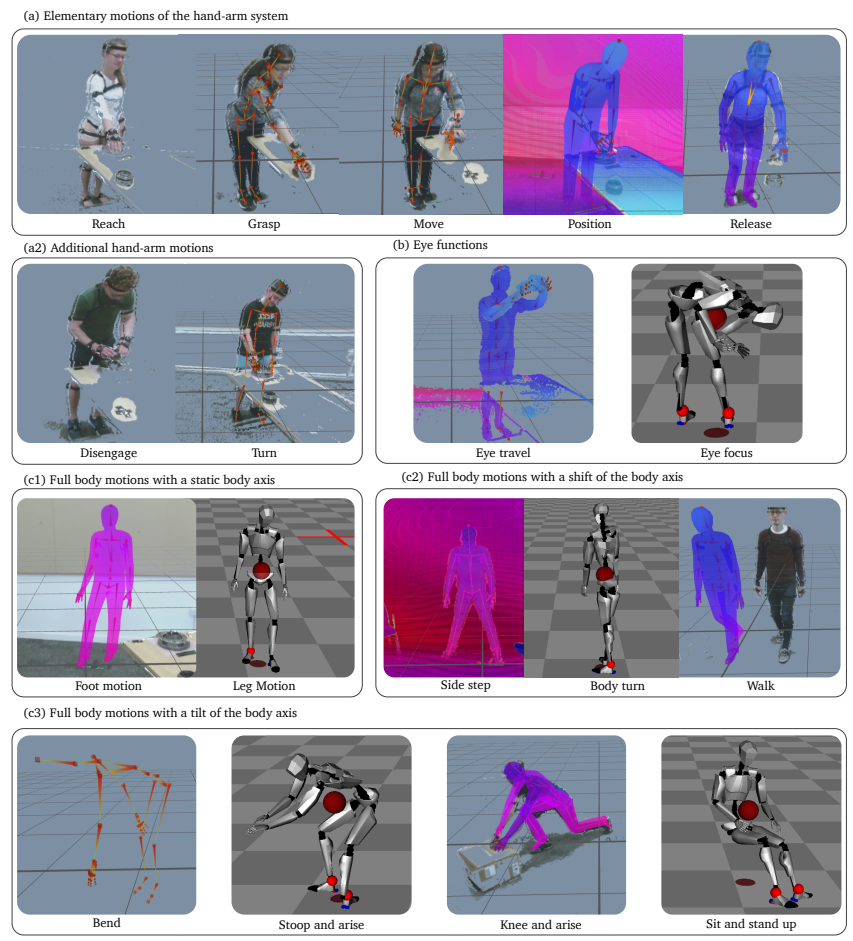

Fig. 4. Exemplary documentation of the 18 activities visualised with a variation of skeleton, depth, pointcloud, RGB(-D), Axis avatar (IMUs), and combinatory representations.

\section{Feature Selection}

Body model features revealed their suitability in HAR for depth as well as IMU data [21]. For the depth data 3D coordinates $(c)$, velocity $(v)$, and acceleration $(a)$ in a cartesian logic were derivable from the raw sensor data. In case of the IMU suit, 3D displacement (Dxyz) and rotation $(R x y z)$ data could be extracted for each skeletal joint. In order to reduce feature dimensionality, we implemented a threestage approach: the first was selecting features of body parts which were continuously trackable by the depth sensor during all activities. In the second phase, features were selected which promised a high contribution to the recognition of an activity due to the involved body parts. Thirdly, PCA was applied to further reduce dimensionality and overfitting. In this framework we compared a 9- versus an 18-body joint configuration, see Figure 5.

\begin{tabular}{c|l}
\hline $\begin{array}{l}\text { Skeletal } \\
\text { joints }\end{array}$ & Skeletal joint terminology \\
\hline 9 & $\begin{array}{l}\text { Head, Neck, Chest, Rshin, Lshin, Rforearm, Lforearm, Rhand, Lhand } \\
\text { Head, Neck, Chest, Center_of_mass, Hip, Rforearm, Lforearm, Rhand, Lhand, Rshin, } \\
\text { Lshin, Rshoulder, Lshoulder, RThigh, LThigh }\end{array}$ \\
\hline
\end{tabular}

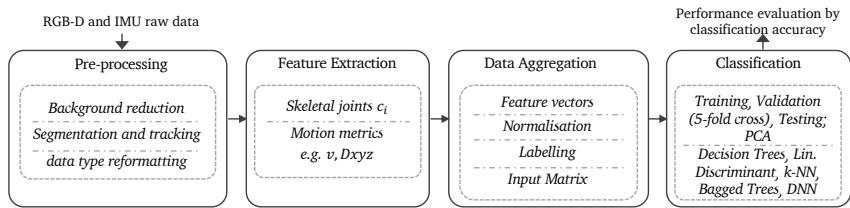

Fig. 6. System architecture for the end-to-end data analysis work stream

\section{RESUlts}

Several subsets of feature combinations and algorithms were tested on the dataset. In the following the $3 \mathrm{D}$ feature set will be referred to as $c_{i}$ with $i=\{9,18\}$ being the number of skeletal body joints (e.g. hip, right hand, left shin). Chosen input modes are characterised by $v$ for velocity in $x y z$ direction (depth data) and Dxyz for the displacement of the IMU data. The classification accuracy was used as the primary decision criteria [9].

\section{A. Comparison of Classification Algorithms}

Firstly, the optimal hyperparameters ( $k$ neighbours, number of splits), and if applicable the optimal number of layers $(w)$, were iteratively derived for all machine learning models before undergoing comparison with other algorithms. After training, all selected algorithms with the different selection of features, a direct comparison can be drawn (see Table II). It can be seen that k-NN and Bagged Trees returned the best classification accuracies for both inertial and visionbased data. The best results with an accuracy of $99.5 \%$ were achieved from Fine k-NN $(k=1)$ on the inertial displacement data using nine body joints. For the Kinect's velocity data, the best result $(79.9 \%)$ was also achieved with the Fine k-NN algorithm. In general, the IMU data enabled higher accuracies for all applied algorithms than the depth data. Linear Discriminant and Decision Trees returned poor results throughout all feature configurations with accuracies below $50 \%$. Moreover, having more features did not improve performance, in most cases it was even lower for the 18 skeletal joint configurations. Deep learning revealed classification accuracies of over $75 \%$ for the inertial data. All DNN models were developed on an established ratio of training, validation, and testing data of $60 \%-20 \%-20 \%$. However, the results for the Kinect data were considerably lower $(<17 \%)$. It can be seen that PCA reduced the initially high accuracy within the Kinect's velocity data for Fine kNN and Bagged Trees. However, PCA only had a moderate decreasing impact on the IMU displacement data.

TABLE II. OVERVIEW OVER THE SELECTED ALGORITHMS' CLASSIFICATION ACCURACY RESULTS FOR THE FOUR MOST PROMISING FEATURE/INPUT MODE CONFIGURATIONS

\begin{tabular}{|c|c|c|c|c|c|}
\hline \multirow[b]{2}{*}{ Classifier } & \multirow[b]{2}{*}{$\begin{array}{l}\text { PCA } \\
X / \sqrt{ }\end{array}$} & \multicolumn{2}{|c|}{ Depth Data } & \multicolumn{2}{|c|}{ IMU Data } \\
\hline & & $\begin{array}{c}\text { Accuracy } \\
c_{9}|v| \text { act }_{18}\end{array}$ & $\begin{array}{c}\text { Accuracy } \\
\mathrm{c}_{18}|v| \text { act }_{18}\end{array}$ & $\begin{array}{c}\text { Accuracy } \\
\mathrm{c}_{9} \mid \text { Dxyz } \mid \text { act }_{18}\end{array}$ & $\begin{array}{c}\text { Accuracy } \\
\mathrm{c}_{18} \mid \text { Dxyz } \mid \text { act }_{18}\end{array}$ \\
\hline $\begin{array}{l}\text { Decision } \\
\text { Tree }\end{array}$ & $\begin{array}{l}x \\
v\end{array}$ & $\begin{array}{l}33.6 \% \\
32.8 \%\end{array}$ & $\begin{array}{l}34.1 \% \\
26.1 \%\end{array}$ & $\begin{array}{l}45.6 \% \\
48.5 \%\end{array}$ & $\begin{array}{l}49.5 \% \\
48.0 \%\end{array}$ \\
\hline Lin. Discr. & $\begin{array}{l}x \\
v\end{array}$ & $\begin{array}{l}16.9 \% \\
16.9 \% \\
\end{array}$ & $\begin{array}{l}24.5 \% \\
12.8 \% \\
\end{array}$ & $\begin{array}{l}39.4 \% \\
14.8 \%\end{array}$ & $\begin{array}{l}55.7 \% \\
14.7 \% \\
\end{array}$ \\
\hline $\begin{array}{l}\text { Fine k- } \\
\text { NN }\end{array}$ & $\begin{array}{l}x \\
v\end{array}$ & $\begin{array}{l}79.9 \% \\
52.1 \%\end{array}$ & $\begin{array}{l}79.6 \% \\
39.7 \%\end{array}$ & $\begin{array}{l}99.5 \% \\
97.0 \%\end{array}$ & $\begin{array}{l}99.4 \% \\
96.8 \%\end{array}$ \\
\hline $\begin{array}{c}\text { Bagged } \\
\text { Tree }\end{array}$ & $\begin{array}{l}x \\
v\end{array}$ & $\begin{array}{l}75.1 \% \\
57.0 \%\end{array}$ & $\begin{array}{l}70.3 \% \\
44.6 \%\end{array}$ & $\begin{array}{l}99.3 \% \\
96.8 \%\end{array}$ & $\begin{array}{l}\mathbf{9 9 . 4} \% \\
96.7 \%\end{array}$ \\
\hline $\begin{array}{c}\text { DNN } \\
(\mathrm{w}=150)\end{array}$ & $x$ & $16.3 \%$ & $13.8 \%$ & $77.0 \%$ & $75.2 \%$ \\
\hline
\end{tabular}

Fig. 5. Selected body joints within the feature extraction process. 


\section{B. Inter-class Similarity}

By consulting the confusion matrix of the Fine k-NN $\left(c_{18}\right.$ $\left.|v| a c t_{18}\right)$ activity 5, 6, 7 (positioning, disengaging, and screwing) and 10 (foot motion) revealed the lowest accuracies. They are thus the least distinctive with regard to the sensor limitations, as they involve fine motor hand or foot motions. In terms of inter-class similarity, it can further be seen that false negatives more often occur within the same MTM activity sub-categories (1-7, 10-14, 15-18), however little confusion occurred between the different categories (see Figure 6).

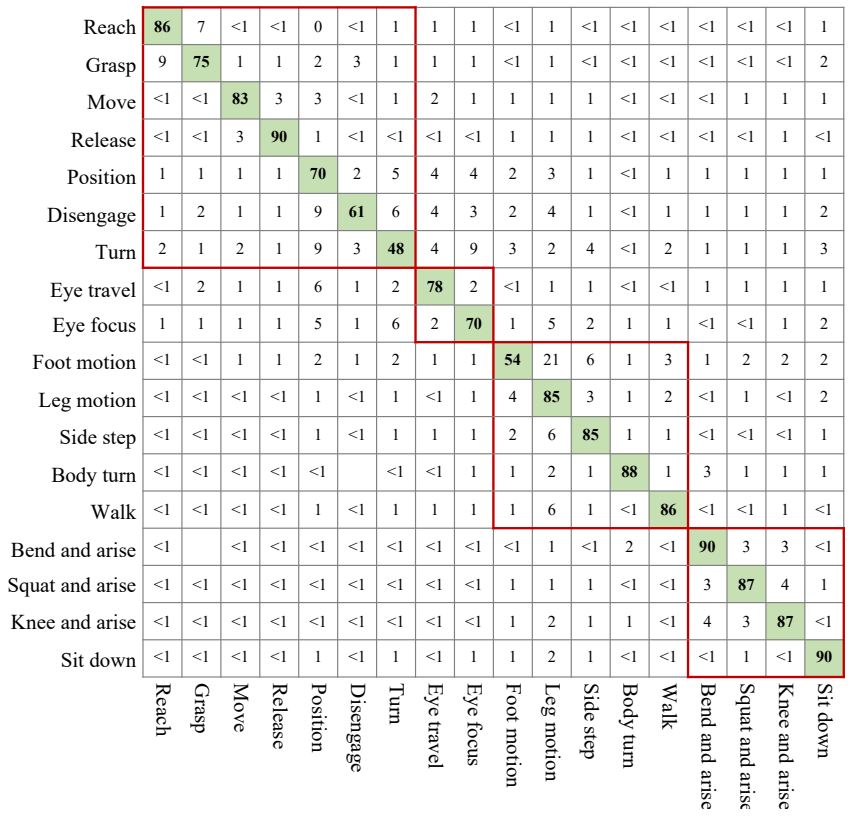

Fig. 7. Confusion matrix for fine k-NN. The green fields display the true positive values (correct predictions), all other represent false negatives. The red squares indicate the MTM task subgroups.

\section{DISCUSSION}

Our main finding was the suitability of MTM-1 as a methodological foundation for the design of context-aware CPAS. This could motivate future research to include standardised work measurement methods into CPAS. Both inertial and RGB-D sensors returned results that are at least equal or better than state-of-the-art approaches. This proved the suitability of our dataset. Lastly, this work contributes to the scientific community by providing the first publicly available dataset of human activities in industrial systems.

\section{A. Sensor-specific and Algorithmic Limitations}

Most noteworthy is the high classification accuracy with k-NN (97.0\%) and Bagged Tree (96.8\%) techniques for the IMU data. The results indicate that it is not necessarily best to use the most advanced, computationally complex algorithms, instead of basic, non-parametric classifiers. As even applying PCA to decrease overfitting revealed high accuracies, the generalisability of our approach could be enhanced for inertial sensors. Noteworthy is also the fact that for both inertial and vision-based sensors a subset of only 9 skeletal joints revealed better results than the use of 18 skeletal joints. This finding aligns with the so-called curseof-dimensionality-theory [29]. However, it might indicate that the full body suit of 17 IMUs is more than sufficient for our intentions. It must be assumed that analysing a subset of
9 body parts is not yet the optimum feature configuration for both sensors, as the features were derived manually. The feature selection could be automated in future research, e.g. by implementing convolutional neural networks [30].

However, it is important to emphasise the difference in recognition performance on the inertial compared to the visual sensor data. The lower classification accuracy results of vision-based sensors $(<80 \%)$ compared to inertial $(>90 \%)$ can be partly explained by the sensor specific limitations of vision-based devices, e.g. occlusion [9]. Especially the body turn or hand-arm motions behind the workbench are prone to occlusion.

Even if inertial sensors provided promising results, magnetic interference might be a major issue within longterm use caused by aggregates on the shop floor. Magnetic interference causes drift which might impair the predictive quality of inertial data. However, first suppliers of inertial sensor suits claim to have full magnetic immunity.

\section{B. Implications for Cyber-Physical Assistance Systems}

Besides technological constraints, human-centric aspects like user experience and acceptance are central to pave the way towards implementing HAR in industrial systems. Reducing the number of wearable sensors, and thus the risk of impairing workers, would be a first step to enhance the applicability. Moreover, inertial and vision-based sensors are obtrusive, novel artefacts in an operator's daily routine that might cause concerns in terms of information privacy. Even if participants were thoroughly briefed and did not report inconvenience, future studies would do well if further examining the long-term acceptance of such systems.

Whether this research's basic proof-of-concept can be extended to an industrial context depends on the ability to close feedback loops in CPPS [31]. Proving the feasibility of recognizing highly similar activities in manufacturing was the first step towards context-awareness. Future studies should investigate how HAR can be optimally integrated in robust industrial applications. Especially the user interface will play a dominant role in the success of CPAS. As an input, the system should receive information about e.g. the sequence of tasks. The system should then monitor the activities in real time and adequately notify the operator if she forgets or insufficiently executes a task.

It appears that our novel HAR approach of building on standardised work measurement methods, in this case MTM, is a step into the right direction. Even highly similar activities, such as reaching and moving, could be recognized with confidence. This ultimately answers how standardised work measurement methods could be integrated in cyber-physical assistance. In the future, it might be promising to extend our approach on aggregated, more complex MTM modules such as MTM-MEK (small-batch production). In addition, our research could be extended to enable ergonomic assessment of industrial tasks with the help of MTM.

\section{CONCLUSIONS}

The contributions of this work regarding the potential of HAR as a foundation for CPAS are threefold. The first contribution is a comprehensive multimodal industrial 
activities dataset containing the scope of the MTM-1 module. The dataset with 19 subjects performing multiple repetitions of 18 activities of three different categories (hand-arm, eye, and full-body motions) exceeds all efforts within an industrial context that could be identified until now. This dataset can be used for future research to assess the potential of sensor fusion approaches, or to optimize feature selection. Secondly, this work presents a contextual use of five machine learning techniques on inertial as well as visual sensors for human activity recognition, applied to manufacturing tasks. Our efforts were the first applying both inertial and vision-based approaches in the same industrial context. By applying both inertial and RGB-D sensors equally high to higher results than state-of-the-art classification accuracies have been achieved. Thirdly, this work is the first that successfully builds an experimental HAR study on an established, standardised work measurement method. Thus, future cyberphysical assistance systems built on this dataset have a greater potential to be implemented into existing standardised industrial environments. In conclusion, the insights gained by our research might indicate that MTM-based, machinelearning-enabled human activity recognition based on inertial and visual sensors is a suitable approach towards the development of context-aware, human-centred CPAS in standardised industrial environments.

\section{REFERENCES}

[1] H. Lasi, P. Fettke, H.-G. Kemper, T. Feld, and M. Hoffmann, "Industry 4.0," Bus. Inf. Syst. Eng., vol. 6, no. 4, pp. 239-242, 2014.

[2] R. Spillner, Einsatz und Planung von Roboterassistenz zur Berücksichtigung von Leistungswandlungen in der Produktion, vol. 296. Herbert Utz Verlag, 2015.

[3] E. M. Frazzon, J. Hartmann, T. Makuschewitz, and B. Scholz-Reiter, "Towards socio-cyber-physical systems in production networks," Procedia Cirp, vol. 7, pp. 49-54, 2013.

[4] B. V. Guerreiro, R. G. Lins, J. Sun, and R. Schmitt, "Definition of Smart Retrofitting: First Steps for a Company to Deploy Aspects of Industry 4.0," in Advances in Manufacturing, A. Hamrol, O. Ciszak, S. Legutko, and M. Jurczyk, Eds. Springer, Cham, 2018.

[5] P. Hold and W. Sihn, "Towards a model to identify the need and the economic efficiency of digital assistance systems in cyber-physical assembly systems." pp. 1-4, 2016.

[6] P. Wang, H. Liu, L. Wang, and R. X. Gao, "Deep learning-based human motion recognition for predictive context-aware human-robot collaboration," CIRP Ann., vol. 67, no. 1, pp. 17-20, 2018.

[7] A. Bannat et al., "Towards optimal worker assistance: a framework for adaptive selection and presentation of assembly instructions," in Proceedings of the 1st international workshop on cognition for technical systems, Cotesys, 2008.

[8] H. Oestreich, T. Töniges, M. Wojtynek, and S. Wrede, "Interactive Learning of Assembly Processes using Digital Assistance," Procedia Manuf., vol. 31, pp. 14-19, 2019.

[9] A. Bulling, U. Blanke, and B. Schiele, "A tutorial on human activity recognition using body-worn inertial sensors," ACM Comput. Surv., vol. 46, no. 3, p. 33, 2014.

[10] J. K. Aggarwal and L. Xia, "Human activity recognition from 3D data: A review," Pattern Recognit. Lett., vol. 48, pp. 70-80, 2014.

[11] O. J. Woodman, "An introduction to inertial navigation," University of Cambridge, Computer Laboratory, 2007.
[12] O. D. Lara and M. A. Labrador, "A survey on human activity recognition using wearable sensors," IEEE Commun. Surv. tutorials, vol. 15, no. 3, pp. 1192-1209, 2012.

[13] A. Avci, S. Bosch, M. Marin-Perianu, R. Marin-Perianu, and P. Havinga, "Activity recognition using inertial sensing for healthcare, wellbeing and sports applications: A survey," in 23th International conference on architecture of computing systems 2010, 2010, pp. 110.

[14] R. B. Fisher and K. Konolige, "Range Sensors," in Handbook of Robotics, B. Siciliano and O. Khatib, Eds. Springer Berlin Heidelberg, 2008, pp. 521-542.

[15] L. Chen, H. Wei, and J. Ferryman, "A survey of human motion analysis using depth imagery," Pattern Recognit. Lett., vol. 34, no. 15, pp. 1995-2006, 2013.

[16] F. Attal, S. Mohammed, M. Dedabrishvili, F. Chamroukhi, L. Oukhellou, and Y. Amirat, "Physical human activity recognition using wearable sensors," Sensors, vol. 15, no. 12, pp. 31314-31338, 2015.

[17] T. Chau, "A review of analytical techniques for gait data. Part 1: fuzzy, statistical and fractal methods," Gait Posture, vol. 13, no. 1, pp. 49-66, 2001.

[18] P. Lukowicz, A. Timm-Giel, M. Lawo, and O. Herzog, "WearIT@work: Toward Real-World Industrial Wearable Computing," IEEE Pervasive Comput., vol. 6, no. 4, pp. 8-13, 2007.

[19] L. Bao and S. S. Intille, "Activity recognition from user-annotated acceleration data," in International conference on pervasive computing, 2004, pp. 1-17.

[20] A. Roitberg, N. Somani, A. Perzylo, M. Rickert, and A. Knoll, "Multimodal Human Activity Recognition for Industrial Manufacturing Processes in Robotic Workcells," pp. 259-266, 2016.

[21] A. Malaisé, P. Maurice, F. Colas, F. Charpillet, and S. Ivaldi, "Activity recognition with multiple wearable sensors for industrial applications," in ACHI 2018-Eleventh International Conference on Advances in Computer-Human Interactions, 2018.

[22] E. Gutenberg, "Grundlagen der Betriebswirtschaftslehre. Erster Band: Die Produktion," Springer Berlin Heidelberg, 1971.

[23] M. F. Zaeh, M. Wiesbeck, S. Stork, and A. Schubö, "A multidimensional measure for determining the complexity of manual assembly operations," Prod. Eng., vol. 3, no. 4-5, p. 489, 2009.

[24] REFA-Bundesverband e.V., "Industrial Engineering. Standardmethoden zur Produktivitätssteigerung und Prozessoptimierung." Carl Hanser Verlag München, Wien, pp. 241247, 2011.

[25] R. Bokranz and K. Landau, Handbuch Industrial Engineering: Produktivitätsmanagement mit MTM. Schäffer-Poeschel, 2012.

[26] J. Chen, J. Qiu, and C. Ahn, "Construction worker's awkward posture recognition through supervised motion tensor decomposition," Autom. Constr., vol. 77, pp. 67-81, 2017.

[27] J. M. Chaquet, E. J. Carmona, and A. Fernández-Caballero, "A survey of video datasets for human action and activity recognition," Comput. Vis. Image Underst., vol. 117, no. 6, pp. 633-659, 2013.

[28] M. Moencks, V. De Silva, J. Roche, and A. Kondoz, “Adaptive Feature Processing for Robust Human Activity Recognition on a Novel MultiModal Dataset," arXiv Prepr. arXiv1901.02858, 2019.

[29] C. M. Bishop, Neural networks for pattern recognition. Oxford university press, 1995.

[30] J. Yang, M. N. Nguyen, P. P. San, X. L. Li, and S. Krishnaswamy, "Deep convolutional neural networks on multichannel time series for human activity recognition," in Twenty-Fourth International Joint Conference on Artificial Intelligence, 2015.

[31] D. Romero, P. Bernus, O. Noran, J. Stahre, and Å. Fast-Berglund, "The operator 4.0: human cyber-physical systems \& adaptive automation towards human-automation symbiosis work systems," in IFIP international conference on advances in production management systems, 2016, pp. 677-686. 